\title{
A SEMIAMPLENESS CRITERION
}

\author{
FLORIN AMBRO
}

\begin{abstract}
We give a criterion for a real divisor to be rational and semiample.
\end{abstract}

\section{INTRODUCTION}

The aim of this paper is to establish the following criterion for the rationality and semiampleness of a nef divisor with real coefficients (we work over an algebraically closed field of characteristic zero):

Theorem 0.1. Let $X$ be a proper nonsingular algebraic variety containing a simple normal crossings $\sum_{l} E_{l}$. Let $B=\sum_{l} b_{l} E_{l}$ and $D=$ $\sum_{l} d_{l} E_{l}$, with $d_{l}, b_{l} \in \mathbb{R}$, be $\mathbb{R}$-divisors on $X$ satisfying the following properties:

(1) $D$ is nef and $r D-\left(K_{X}+B\right)$ is nef and big for some $r \in \mathbb{Q}$.

(2) $b_{l}<1$ for every $l$.

(3) For every integer $j \geq 1$, the following inclusion holds

$$
H^{0}\left(X, \sum_{l}\left\lceil j d_{l}-b_{l}\right\rceil E_{l}\right) \subseteq H^{0}\left(X, \sum_{l}\left\lfloor j d_{l}\right\rfloor E_{l}\right) .
$$

For a real number $d,\lfloor d\rfloor$ and $\lceil d\rceil$ are the largest and smallest integers, respectively, such that $\lfloor d\rfloor \leq d \leq\lceil d\rceil$.

Then there exists a positive integer $m$ such that $m d_{l} \in \mathbb{Z}$ for every $l$, and the linear system $|m D|$ is base point free.

This criterion is also valid for singular varieties, being birational in nature (see Theorem 2.1). It is a generalization of the Base Point Free Theorem (Kawamata-Shokurov [1), and it also extends the known fact that the existence of the Cutkosky-Kawamata-Moriwaki decomposition for a $\log$ canonical divisor of general type implies the finite generation of the log canonical ring (Kawamata [2]).

\footnotetext{
${ }^{1}$ This work was supported through a Twenty-First Century COE Kyoto Mathematics Fellowship.

${ }^{2} 1991$ Mathematics Subject Classification. Primary: 14C20, 14E30. Secondary: $14 \mathrm{~N} 30$.
} 
Our interest in such a criterion lies in its connection to the finite generation of Shokurov's FGA-algebras ([5], Conjecture 4.39). Shokurov has recently reduced the existence of $\log$ flips in dimension $d+1$ to the Log Minimal Model Program in dimension $d$, and the finite generation of $d$-dimensional FGA-algebras ([5], Corollary 1.5). Thus, provided that FGA-algebras are finitely generated, and this may be established even assuming the validity of the Log Minimal Model Program in the same dimension, this would give an inductive proof of the existence of $\log$ flips. The finite generation of FGA-algebras is known in dimension one and two ([5], Main Theorem 1.7).

For the purpose of this introduction, an FGA-algebra is an $\mathbb{N}$-graded sub-algebra of a divisorial algebra

$$
\mathcal{L} \subseteq \bigoplus_{i=0}^{\infty} H^{0}(X, i D)
$$

where $D$ is some divisor on a Fano variety $X$, such that $\mathcal{L}$ is asymptotically saturated with respect to $X$. More precisely, the normalization of any such sub-algebra admits a representation

$$
\overline{\mathcal{L}}=\bigoplus_{i=0}^{\infty} H^{0}\left(X_{i}, M_{i}\right),
$$

where each $M_{i}$ is a free Cartier divisor on some resolution of singularities $\mu_{i}: X_{i} \rightarrow X$ such that both divisors $K_{X_{i}}-\mu_{i}^{*}\left(K_{X}\right)$ and $M_{i}$ are supported by a simple normal crossings divisor on $X_{i}$. Then asymptotic saturation means that the following inclusions hold, for every $i, j \geq 1$ :

$$
H^{0}\left(X_{i},\left\lceil K_{X_{i}}-\mu_{i}^{*}\left(K_{X}\right)+j \frac{M_{i}}{i}\right\rceil\right) \subseteq H^{0}\left(X_{j}, M_{j}\right)
$$

FGA-algebras are not well understood at the moment, the main difficulty being in dealing with the a priori infinitely many models $X_{i}$. The successful two dimensional approach to FGA-algebras is based on the Canonical Confinement Conjecture ([5], Conjecture 6.14). Alternatively, based on the experience from the proof of the Base Point Free Theorem, Shokurov suggested that an inductive method may be possible in a larger class of algebras: $X$ may no longer be a Fano variety, but it should be related to $\mathcal{L}$ by some kind of adjoint-type property ([5], Remark 4.40.3). As an application of Theorem 0.1] we give two such examples of adjoint-type properties which are sufficient for finite generation:

Theorem 0.2. Let $X$ be a proper nonsingular algebraic variety containing a simple normal crossings divisor $\sum_{l} E_{l}$. Let $D=\sum_{l} d_{l} E_{l}$ be 
a nef a big $\mathbb{R}$-divisor, with associated divisorial algebra

$$
\mathcal{R}_{X}(D)=\bigoplus_{i=0}^{\infty} H^{0}(X, i D) .
$$

Assume that there exists an $\mathbb{R}$-divisor $B=\sum_{l} b_{l} E_{l}$, with $b_{l}<1$ for every $l$, such that

(1) $\mathcal{R}_{X}(D)$ is asymptotically saturated with respect to $(X, B)$.

(2) The $\mathbb{R}$-divisor $r D-\left(K_{X}+B\right)$ is nef, for some $r \in \mathbb{Q}$.

Then $\mathcal{R}_{X}(D)$ is a finitely generated algebra.

Theorem 0.3. Let $X$ be proper nonsingular algebraic variety containing a simple normal crossings divisor $\sum_{l} E_{l}$. Let $M_{i}=\sum_{l} m_{i, l} E_{l}$ be divisors on $X$, for $i \geq 0$, satisfying the following properties:

(i) The linear system $\left|M_{i}\right|$ is base point free, for every $i$.

(ii) $M_{0}=0, M_{i}+M_{j} \leq M_{i+j}$ for every $i, j$.

(iii) The limit $d_{l}=\lim _{i \rightarrow \infty} \frac{1}{i} m_{i, l}$ exists, for every $l$. Set $D=$ $\sum_{l} d_{l} E_{l}$

The sequence $\left(M_{i}\right)_{i \geq 0}$ defines an algebra

$$
\mathcal{L}=\bigoplus_{i=0}^{\infty} H^{0}\left(X, M_{i}\right) \subseteq \mathcal{R}_{X}(D)
$$

Assume moreover that there exists an $\mathbb{R}$-divisor $B=\sum_{l} b_{l} E_{l}$, with $b_{l}<1$ for every $l$, such that

(1) $\mathcal{L}$ is asymptotically saturated with respect to $(X, B)$. Equivalently, $H^{0}\left(X, \sum_{l}\left\lceil j \frac{m_{i, l}}{i}-b_{l}\right\rceil E_{l}\right) \subseteq H^{0}\left(X, M_{j}\right)$ for every $i, j \geq$ 1.

(2) The $\mathbb{R}$-divisor $r D-\left(K_{X}+B\right)$ is nef and big, for some $r \in \mathbb{Q}$. Then $\mathcal{L}$ is a finitely generated algebra.

The paper is organized as follows. In Section 1, we collect some basic definitions and properties on log pairs, b-divisors and functional algebras. In Section 2, we establish the semiampleness criterion. The proof is quite similar to that of the Base Point Free Theorem, and we use a nonvanishing result for real divisors due to Kawamata (2], Theorem 3 ). In Section 3, we apply this criterion to the finite generation of some functional algebras.

\section{Preliminary}

A variety is a reduced and irreducible separable scheme of finite type, defined over an algebraically closed field $k$ of characteristic zero. 
A contraction is a proper morphism of normal varieties $f: X \rightarrow Y$ with $\mathcal{O}_{Y}=f_{*} \mathcal{O}_{X}$.

1-A. Divisors. Let $X$ a normal variety. A Weil divisor on $X$ is an element of the free abelian group $\operatorname{WDiv}(X)$ generated by codimension one cycles on $X$. A non-zero rational function $a \in k(X)^{\times}$defines the principal Weil divisor

$$
(a)=\sum_{\operatorname{codim}(P, X)=1} \operatorname{mult}_{P}(a) P .
$$

Let $L \in\{\mathbb{Z}, \mathbb{Q}, \mathbb{R}\}$. An $L$-Weil divisor is an element of

$$
\operatorname{WDiv}(X)_{L}:=\operatorname{WDiv}(X) \otimes_{\mathbb{Z}} L .
$$

We say that $D_{1}, D_{2} \in \mathrm{WDiv}(X)_{\mathbb{R}}$ are L-linearly equivalent, denoted $D_{1} \sim_{L} D_{2}$, if there exist finitely many $q_{i} \in L$ and $a_{i} \in k(X)^{\times}$such that $D_{1}-D_{2}=\sum_{i} q_{i}\left(a_{i}\right)$. We say that $D \in \mathrm{WDiv}(X)_{L}$ is $L$-Cartier if $D \sim_{L} 0$ in a neighborhood of each point of $X$. We denote by

$$
\operatorname{CDiv}(X)_{L} \subset \operatorname{WDiv}(X)_{L}
$$

the subgroup of $L$-Cartier $L$-Weil divisors. To each $D \in \operatorname{WDiv}(X)_{\mathbb{R}}$ we can associate a coherent $\mathcal{O}_{X}$-sub-module $\mathcal{O}_{X}(D)$ of the constant sheaf of rational functions $k(X)$ on $X$, whose sections on open subsets $U$ of $X$ are given by

$$
H^{0}\left(U, \mathcal{O}_{X}(D)\right)=\left\{a \in k(X)^{\times} ;\left.((a)+D)\right|_{U} \geq 0\right\} \cup\{0\} .
$$

For $D \in \mathrm{WDiv}(X)_{\mathbb{R}}$, its round down $\lfloor D\rfloor$, round up $\lceil D\rceil$, fractional part $\{D\}$ are defined componentwise (for $d \in \mathbb{R},\lfloor d\rfloor$ and $\lceil d\rceil$ are the unique integers in the half-open intervals $(d-1, d]$ and $[d, d+1)$, respectively, and $\{d\}=d-\lfloor d\rfloor)$.

Let $\pi: X \rightarrow S$ be a proper surjective morphism. We say that $D \in$ $\operatorname{WDiv}(X)_{\mathbb{R}}$ is

(i) free $/ S$ if $D$ is Cartier and the natural map $\pi^{*} \pi_{*} \mathcal{O}_{X}(D) \rightarrow$ $\mathcal{O}_{X}(D)$ is surjective.

(ii) $n e f / S$ if $D$ is $\mathbb{R}$-Cartier and $D \cdot C \geq 0$ for every proper curve $C \subset X$ such that $\pi(C)$ is a point.

(iii) ample/S if $\pi$ is projective and the numerical class of $D$ belongs to the real cone generated by the numerical classes of $\pi$-ample Cartier divisors.

(iv) semiample/S if there exists a contraction $f: X \rightarrow Y$, defined over $S$, and an ample/S $\mathbb{R}$-divisor $H$ on $Y$ such that $D \sim_{\mathbb{R}}$ $f^{*} H$. If $D$ is rational, this is equivalent to the existence of a positive integer $m$ such that $m D$ is free/S. 
(v) $\mathrm{big} / \mathrm{S}$ if there exists $C>0$ such that $\operatorname{rank} \pi_{*} \mathcal{O}_{X}(m D) \geq C m^{d}$ for $m$ sufficiently large and divisible, where $d$ is the dimension of the general fiber of $\pi$.

1-B. B-divisors. (V.V. Shokurov 4]). Let $X$ be a normal variety. The group of $b$-divisors of $X$ is defined as the projective limit

$$
\operatorname{BDiv}(X)=\underset{Y \rightarrow X}{\operatorname{proj}} \lim \operatorname{Wiv}(Y),
$$

where the index set is the set of isomorphism classes of birational contractions $Y \rightarrow X$, with the following order: $Y_{1} \geq Y_{2}$ if the induced proper birational map $g: Y_{1} \rightarrow Y_{2}$ is a morphism. In this case, we have a natural push-forward homomorphism $g_{*}: \operatorname{WDiv}\left(Y_{1}\right) \rightarrow \operatorname{WDiv}\left(Y_{2}\right)$. Note that given two birational contractions $Y_{1} \rightarrow X$ and $Y_{2} \rightarrow X$, the normalization of the graph of the induced birational map $Y_{1} \rightarrow Y_{2}$ defines a third birational contraction $Y_{3} \rightarrow X$ with $Y_{3} \geq Y_{1}$ and $Y_{3} \geq Y_{2}$.

A prime b-divisor $E$ of $X$ is obtained as follows: let $\mu: Y \rightarrow X$ be a birational contraction, and let $E$ be a prime divisor on $Y$. For another birational contraction $Y^{\prime} \rightarrow X$, let $g: Y \rightarrow Y^{\prime}$ be the induced rational map. If $g$ induces an isomorphism between the generic point of $E$ and the generic point of a prime divisor $E^{\prime}$ on $Y^{\prime}$, we set $\mathbf{D}_{Y^{\prime}}=E^{\prime}$. Otherwise, set $\mathbf{D}_{Y^{\prime}}=0$. The family $\left\{\mathbf{D}_{Y^{\prime}}\right\}$ is a prime b-divisor of $X$, denoted by $E$. The center of $E$ on $X$, denoted by $c_{X}(E)$, is the closed subset $\mu(E)$ of $X$. It is clear that prime b-divisors of $X$ are in one-toone correspondence with geometric (discrete) valuations of $k(X)$ which have non-empty center on $X$. In this terminology, a b-divisor of $X$ is a $\mathbb{Z}$-valued function on the set of all prime b-divisors of $X$, denoted as a formal sum

$$
\mathbf{D}=\sum_{E} \operatorname{mult}_{E}(\mathbf{D}) E
$$

such that the sum has finite support on some (hence any) birational model of $X$.

A proper birational map $\Phi: X_{1} \rightarrow X_{2}$ (i.e., the normalization of the graph of $\Phi$ induces birational contractions to $X_{1}$ and $\left.X_{2}\right)$, induces a push-forward isomorphism $\Phi_{*}: \operatorname{BDiv}\left(X_{1}\right) \stackrel{\sim}{\rightarrow} \operatorname{BDiv}\left(X_{2}\right)$.

For $L \in\{\mathbb{Z}, \mathbb{Q}, \mathbb{R}\}$, an $L$-b-divisors of $X$ is an element of

$$
\operatorname{BDiv}(X)_{L}:=\operatorname{BDiv}(X) \otimes_{\mathbb{Z}} L .
$$

Examples 1.1. (1) A rational function $a \in k(X)^{\times}$defines the following b-divisor of $X$

$$
\overline{(a)}=\left\{\left(f^{*} a\right)\right\}_{f: Y \rightarrow X} .
$$


(2) Let $Y \rightarrow X$ be a birational contraction and let $D \in \operatorname{CDiv}(Y)_{\mathbb{R}}$. The family $\bar{D}=\left\{f^{*} D\right\}_{f: Y^{\prime} \rightarrow Y}$ is an $\mathbb{R}$-b-divisor of $X$, called the Cartier closure of $D$.

(3) Let $\omega \in \Omega_{k(X) / k}^{\operatorname{dim}(X)}$ be a top rational differential form of $X$. The associated family of Weil divisors $\mathbf{K}=\left\{\left(f^{*} \omega\right)\right\}_{f: Y \rightarrow X}$ is called the canonical b-divisor of $X$. It is uniquely defined up to linear equivalence.

Let $\mathbf{D}$ be an $\mathbb{R}$-b-divisor of $X$. The round up $\lceil\mathbf{D}\rceil \in \operatorname{BDiv}(X)$ is defined componentwise. The restriction of $\mathbf{D}$ to an open subset $U \subset X$ is a well defined $\mathbb{R}$-b-divisor of $U$, denoted by $\left.\mathbf{D}\right|_{U}$. Also, $\mathbf{D}$ defines an $\mathcal{O}_{X}$-module $\mathcal{O}_{X}(\mathbf{D})$ whose sections on an open subset $U \subseteq X$ are given by

$$
H^{0}\left(U, \mathcal{O}_{X}(\mathbf{D})\right)=\left\{a \in k(X)^{\times} ;\left.(\overline{(a)}+\mathbf{D})\right|_{U} \geq 0\right\} \cup\{0\} .
$$

An $\mathbb{R}$-b-divisor $\mathbf{D}$ of $X$ is called $L$-b-Cartier if there exists a birational contraction $f: X^{\prime} \rightarrow X$ such that $\mathbf{D}_{X^{\prime}}$ is $L$-Cartier and $\mathbf{D}=\overline{\mathbf{D}_{X^{\prime}}}$. Note that $\mathcal{O}_{X}(\mathbf{D})=f_{*} \mathcal{O}_{X^{\prime}}\left(\mathbf{D}_{X^{\prime}}\right)$ in this case, in particular $\mathcal{O}_{X}(\mathbf{D})$ is a coherent $\mathcal{O}_{X}$-module.

Let $\pi: X \rightarrow S$ be a proper morphism. An $\mathbb{R}$-b-divisor $\mathbf{D}$ of $X$ is called b-free/S (b-nef/S, b-semiample/S, b-nef and big/S) if there exists a birational contraction $X^{\prime} \rightarrow X$ such that $\mathbf{D}=\overline{\mathbf{D}_{X^{\prime}}}$ and $\mathbf{D}_{X^{\prime}}$ is free/S (nef/S, semiample/S, nef/S and big/S), where $X^{\prime} \rightarrow S$ is the induced proper morphism.

Lemma 1.2 ([5], Proposition 4.15). Let $\pi: X \rightarrow S$ be a contraction. Let $k(X)$ be the constant sheaf of rational functions on $X$ and let $\mathcal{L} \subset$ $\pi_{*} k(X)$ be a non-zero coherent $\mathcal{O}_{X}$-module. Then there exists a unique $b$-free/S b-divisor $\mathbf{M}$ of $X$ such that

(i) $\mathcal{L} \subseteq \pi_{*} \mathcal{O}_{X}(\mathbf{M})$.

(ii) If $\overline{\mathbf{D}} \in \operatorname{BDiv}(X)_{\mathbb{R}}$ and $\mathcal{L} \subseteq \pi_{*} \mathcal{O}_{X}(\mathbf{D})$, then $\mathbf{M} \leq \mathbf{D}$.

$W e$ say that $\mathbf{M}$ is the mobile b-divisor of $\mathcal{L}$.

Sketch of proof. Assume first that $S$ is affine, so that $\mathcal{L}$ is generated by finitely many global sections $a_{1}, \ldots, a_{p}$. The b-divisor $\mathbf{M}$ is defined by

$$
\mathbf{M}=\sup \left\{-\overline{(a)} ; a \in H^{0}(S, \mathcal{L}) \backslash 0\right\}=\max _{i=1}^{p}\left(-\overline{\left(a_{i}\right)}\right)
$$

In general, we may cover $S$ with finitely many affine open subsets $S_{\alpha}$, and the b-divisors $\mathbf{M}_{\alpha}$ of $\pi^{-1}\left(S_{\alpha}\right)$ constructed above glue to a b-divisor $\mathbf{M} \in \operatorname{BDiv}(X)$, which clearly satisfies (i) and (ii).

To show that $\mathbf{M}$ is b-free/ $S$, assume again that $S$ is affine. By the definition of $\mathbf{M}_{X}$, the linear system

$$
\left\{(a)+\mathbf{M}_{X} ; a \in H^{0}(S, \mathcal{L}) \backslash 0\right\}
$$


has no fixed components. By Hironaka, there exists a birational contraction $\mu: Y \rightarrow X$ such that the induced linear system

$$
\left\{\left(\mu^{*} a\right)+\mathbf{M}_{Y} ; a \in H^{0}(S, \mathcal{L}) \backslash 0\right\}
$$

has no fixed points. Then $\mathbf{M}_{Y}$ is free $/ S$ and $\mathbf{M}=\overline{\mathbf{M}_{Y}}$.

Lemma 1.3. Let $\pi: X \rightarrow S$ be a contraction. Let $\mathbf{D} \in \operatorname{BDiv}(X)_{\mathbb{R}}$ such that $\mathbf{D}_{X} \leq 0$ and $\mathbf{D}$ is b-nef/S. Then:

(1) $\mathbf{D} \leq 0$.

(2) There exists a closed subset $S^{0} \varsubsetneqq S$ such that for every prime $b$-divisor $E$ of $X, \operatorname{mult}_{E}(\mathbf{D}) \neq 0$ if and only if $\pi\left(c_{X}(E)\right) \subseteq$ $S^{0}$.

Proof. (1) Let $Y \rightarrow X$ be a birational contraction such that $\mathbf{D}=\overline{\mathbf{D}_{Y}}$. Since $\mathbf{D}_{Y}$ is nef $/ S$, it is also nef $/ X$. By the Negativity Lemma [3, 1.1], we obtain $\mathbf{D}_{Y} \leq 0$. Therefore $\mathbf{D}=\overline{\mathbf{D}_{Y}} \leq 0$.

(2) Let $Y \rightarrow X$ be a birational contraction such that $\mathbf{D}=\overline{\mathbf{D}_{Y}}$, and let $\nu: Y \rightarrow S$ be the induced contraction. The restriction of the $\mathbb{R}$-divisor $-\mathbf{D}_{Y}$ to the general fibre of $\nu$ is zero, being anti-nef and effective. Therefore

$$
S^{0}=\nu\left(\operatorname{Supp}\left(\mathbf{D}_{Y}\right)\right)
$$

is a closed subset of $S$ and $S^{0} \neq S$. Property (2) is equivalent to the following equality of sets

$$
\operatorname{Supp}\left(\mathbf{D}_{Y}\right)=\nu^{-1}\left(S^{0}\right) .
$$

The inclusion $\operatorname{Supp}\left(\mathbf{D}_{Y}\right) \subseteq \nu^{-1}\left(S^{0}\right)$ is clear. For the converse, fix a point $P \in S^{0}$ and assume by contradiction that $\nu^{-1}(P)$ is not included in $\operatorname{Supp}\left(\mathbf{D}_{Y}\right)$. We may replace $Y$ by a resolution of singularities such that $\nu^{-1}(P)$ is the union of finitely many prime Cartier divisors $E_{1}, \ldots, E_{n}$ on $Y$. By assumption, we have $\operatorname{mult}_{E_{1}}\left(\mathbf{D}_{Y}\right)=0$. The fiber $\nu^{-1}(P)=\bigcup_{i=1}^{n} E_{i}$ is connected, hence after a possible reordering, we may assume that $E_{j}$ intersects $E_{1} \cup \cdots \cup E_{j-1}$, for every $2 \leq j \leq n$.

Assume by induction that mult $_{E_{i}}\left(\mathbf{D}_{Y}\right)=0$ for $i \leq j-1$. There exists a proper curve $C \subset \bigcup_{i=1}^{j-1} E_{i}$ such that $C \cap E_{j} \neq \emptyset$ and $C \nsubseteq \operatorname{Supp}\left(\mathbf{D}_{Y}\right)$. Since $-\mathbf{D}_{Y}$ is effective and $\mathbf{D}_{Y} \cdot C \geq 0$, we infer mult $E_{j}\left(\mathbf{D}_{Y}\right)=0$. By induction, we obtain $\operatorname{mult}_{E_{j}}\left(\mathbf{D}_{Y}\right)=0$ for $1 \leq j \leq n$. However, $P \in S^{0}$ implies that $\operatorname{Supp}\left(\mathbf{D}_{Y}\right) \cap \nu^{-1}(P) \neq \emptyset$. In particular, there exists a proper curve $C \subseteq \nu^{-1}(P)$ such that $C \nsubseteq \operatorname{Supp}\left(\mathbf{D}_{Y}\right)$ and $C \cap$ $\operatorname{Supp}\left(\mathbf{D}_{Y}\right) \neq \emptyset$. Therefore $\mathbf{D}_{Y} \cdot C<0$. On the other hand, $\mathbf{D}_{Y}$ is nef $/ S$, hence $\mathbf{D}_{Y} \cdot C \geq 0$. Contradiction.

Lemma 1.4. Let $X \rightarrow S$ be a contraction, and let $\mathbf{D} \leq \mathbf{H}$ be $\mathbb{R}-b$ divisors of $X$ such that $\mathbf{D}$ is b-nef/S and $\mathbf{H}$ is $\mathbb{R}$-b-semiample/S, that 
is there exists rational contraction over $S$

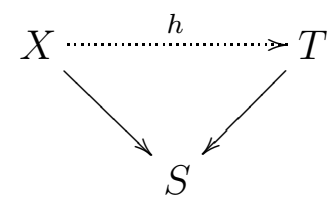

and an ample $/ S \mathbb{R}$-divisor $A$ on $T$ such that $\mathbf{H} \sim_{\mathbb{R}} \overline{h^{*}(A)}$. If $\mathbf{D}=\mathbf{H}$ over a neighborhood of a closed subset $X_{0} \subset X$, then $\mathbf{D}=\mathbf{H}$ over a neighborhood of $h\left(X_{0}\right) \subset T$.

Proof. We may replace $X$ by a higher birational model so that $h$ becomes a morphism. By assumption, $\mathbf{D}-\mathbf{H} \leq 0$ is b-nef $/ T$. By Lemma 1.3. there exists a closed subset $T^{0} \subset T$ such that mult ${ }_{E}(\mathbf{D}-$ $\mathbf{H}) \neq 0$ if and only if $c_{X}(E) \subseteq h^{-1}\left(T^{0}\right)$, for every prime b-divisor $E$ of $X$. By assumption, there exists an open set $U \supset X_{0}$ such that mult $_{E}(\mathbf{D}-\mathbf{H})=0$ for every prime b-divisor $E$ of $X$ with $c_{X}(E) \cap U \neq \emptyset$. Therefore $U \subseteq X \backslash h^{-1}\left(T^{0}\right)$. In particular, $X_{0} \cap h^{-1}\left(T^{0}\right)=\emptyset$. Therefore $V=T \backslash T^{0}$ is an open neighborhood of $h\left(X_{0}\right)$, and $\left.\mathbf{D}\right|_{h^{-1}(V)}=$ $\left.\mathbf{H}\right|_{h^{-1}(V)}$.

1-C. Log pairs. A $\log \operatorname{pair}(X, B)$ is a normal variety $X$ endowed with an $\mathbb{R}$-Weil divisor $B$ such that $K+B$ is $\mathbb{R}$-Cartier. A log variety is a $\log$ pair $(X, B)$ such that $B$ is effective.

Let $(X, B)$ be a log pair and let $\mu: Y \rightarrow X$ be a birational contraction. Choose a top rational differential form $\omega \in \Omega_{k(X) / k}^{\operatorname{dim}(X)}$ with $K=(\omega)$. The Weil divisor $K_{Y}=\left(\mu^{*} \omega\right)$ is a canonical divisor of $Y$, and there exists a unique $\mathbb{R}$-Weil divisor $B_{Y}$ on $Y$ satisfying the adjunction formula

$$
\mu^{*}(K+B)=K_{Y}+B_{Y} .
$$

We say that $\left(Y, B_{Y}\right)$ is the crepant $\log$ pair structure induced by $(X, B)$ via $\mu$. It is independent of the choice of $\omega$ and in fact it is independent of the choice of $K$ in its linear equivalence class. There exists a unique $\mathbb{R}$-b-divisor $\mathbf{A}(X, B)$ of $X$, called the discrepancy $\mathbb{R}$-b-divisor of the log pair $(X, B)$, whose trace on birational modifications $Y \rightarrow X$ is

$$
\mathbf{A}(X, B)_{Y}=-B_{Y} .
$$

A log pair $(X, B)$ is said to have Kawamata log terminal singularities if mult $_{E}(\mathbf{A}(X, B))>-1$ for every geometric valuation $E$ of $X$. We denote by $\operatorname{LCS}(X, B)$ the largest closed subset of $X$ on whose complement $(X, B)$ has Kawamata log terminal singularities.

For a log pair $(X, B)$, the $\mathcal{O}_{X}$-module $\mathcal{O}_{X}(\lceil\mathbf{A}(X, B)\rceil)$ is coherent. Indeed, let $\mu: Y \rightarrow X$ be a resolution of singularities such that $B_{Y}=$ 
$\sum_{l} b_{l} E_{l}$ and $\sum_{l} E_{l}$ is a simple normal crossings divisor. Then (see the proof of [5], Proposition 4.46)

$$
\mathcal{O}_{X}(\lceil\mathbf{A}(X, B)\rceil)=\mu_{*} \mathcal{O}_{Y}\left(\left\lceil-B_{Y}\right\rceil\right) .
$$

Also, $\operatorname{LCS}(X, B)=\mu\left(\operatorname{LCS}\left(Y, B_{Y}\right)\right)$ and $\operatorname{LCS}\left(Y, B_{Y}\right)=\bigcup_{b_{l} \geq 1} E_{l}$.

1-D. Functional algebras. (V.V. Shokurov [5]) Let $\pi: X \rightarrow S$ be a contraction. A functional algebra of $X / S$ is an $\mathbb{N}$-graded $\mathcal{O}_{S}$-subalgebra

$$
\mathcal{L} \subset \bigoplus_{i=0}^{\infty} \pi_{*} k(X)
$$

such that $\mathcal{L}_{0}=\mathcal{O}_{S}$ and $\mathcal{L}_{i}$ is a coherent $\mathcal{O}_{S}$-module for every $i \geq 1$. Here, $k(X)$ is the constant sheaf of rational functions on $X$, and the multiplication in $\bigoplus_{i=0}^{\infty} \pi_{*} k(X)$ is induced by that of $k(X)$.

Denote by $\mathbb{N}(\mathcal{L})$ the semigroup of positive integers $i$ with $\mathcal{L}_{i} \neq 0$. For $i \in \mathbb{N}(\mathcal{L})$, let $\mathbf{M}_{i}$ be the mobile b-divisor of $\mathcal{L}_{i}$ (see Lemma 1.2) and set $\mathbf{D}_{i}=\mathbf{M}_{i} / i$. By construction, $\mathbf{M}_{i}$ is b-free $/ S$ and $\mathbf{D}_{i}$ is a bsemiample/S $\mathbb{Q}$-b-divisor. The sequences $\left(\mathbf{M}_{i}\right)_{i \in \mathbb{N}(\mathcal{L})}$ and $\left(\mathbf{D}_{i}\right)_{i \in \mathbb{N}(\mathcal{L})}$ are called the mobile sequence and characteristic sequence of $\mathcal{L}$. Since $\mathcal{L}$ is an algebra, the following properties hold:

(i) $\mathbf{M}_{i}+\mathbf{M}_{j} \leq \mathbf{M}_{i+j}$.

(ii) $\mathbf{M}_{0}=0$.

In particular, $\bigoplus_{i \in\{0\} \cup \mathbb{N}(\mathcal{L})} \pi_{*} \mathcal{O}_{X}\left(\mathbf{M}_{i}\right)$ is a functional algebra of $X / S$, which is the normalization of $\mathcal{L}$ ([5], Proposition 4.15).

The functional algebra $\mathcal{L}$, is called bounded if the supremum

$$
\mathbf{D}=\sup _{i \in \mathbb{N}(\mathcal{L})} \mathbf{D}_{i}
$$

is an $\mathbb{R}$-b-divisor of $X$, called the characteristic limit of $\mathcal{L}$. In this case, the convexity property (i) implies that the supremum is a limit

$$
\mathbf{D}=\lim _{\mathbb{N}(\mathcal{L}) \ni i \rightarrow \infty} \mathbf{D}_{i} .
$$

The $\mathcal{O}_{S}$-algebra $\mathcal{L}$ is finitely generated if and only if $\mathbf{D}_{i}=\mathbf{D}$ for some $i \in \mathbb{N}(\mathcal{L})([5]$, Theorem 4.28).

Let $\mathbf{A} \in \operatorname{BDiv}(X)_{\mathbb{R}}$. The functional algebra $\mathcal{L}$ is asymptotically $\mathbf{A}$ saturated if there exists a positive integer $I$ such that $\mathcal{L}_{i} \neq 0$ for $I \mid i$, and the following inclusion holds for $I \mid i, j$ :

$$
\pi_{*} \mathcal{O}_{X}\left(\left\lceil\mathbf{A}+j \mathbf{D}_{i}\right\rceil\right) \subseteq \pi_{*} \mathcal{O}_{X}\left(\mathbf{M}_{j}\right)
$$

We say that $\mathcal{L}$ is asymptotically saturated with respect to a log pair $(X, B)$ if $\mathcal{L}$ is asymptotically $\mathbf{A}(X, B)$-saturated.

Example 1.5. Let $\pi: X \rightarrow S$ be a contraction. 
(1) Let $\mathbf{D}$ be an $\mathbb{R}$-b-divisor of $X$. Then

$$
\mathcal{R}_{X / S}(\mathbf{D})=\bigoplus_{i=0}^{\infty} \pi_{*} \mathcal{O}_{X}(i \mathbf{D})
$$

is a bounded functional algebra of $X / S$.

(2) Let $(X, B)$ be a $\log$ variety structure on $X$ and let $D \in$ $\operatorname{CDiv}(X)_{\mathbb{Q}}$. Then the normal bounded functional algebra of $X / S$

$$
\mathcal{R}_{X / S}(D)=\bigoplus_{i=0}^{\infty} \pi_{*} \mathcal{O}_{X}(i D)
$$

is asymptotically $\mathbf{A}(X, B)$-saturated.

1-E. Kawamata's Nonvanishing. For a real number $r$, the round off $\langle r\rangle$ is defined as the unique integer lying in the unit interval $[r-$ $1 / 2, r+1 / 2)$. The following properties are easy to check:

(1) $\lfloor r\rfloor \leq\langle r\rangle \leq\lceil r\rceil$.

(2) $\langle-r\rangle=-\langle r\rangle$ unless $\{r\}=\frac{1}{2}$.

(3) $\langle r\rangle=0$ for $r \in\left(-\frac{1}{2}, \frac{1}{2}\right]$.

Lemma 1.6. 2] Let $x \in \mathbb{R}^{d}$, let $I$ be a positive integer and let $\epsilon>0$. Then there exists a positive multiple $m$ of $I$ such that $m x_{1} \leq\left\langle m x_{1}\right\rangle$ and $\max _{i=1}^{d}\left|m x_{i}-\left\langle m x_{i}\right\rangle\right|<\epsilon$.

For an $\mathbb{R}$-divisor $D$ on a normal variety $X$, the round off $\langle D\rangle$ is defined componentwise. Also, the absolute value of $D$ is defined by

$$
\|D\|=\max \left\{\left|\operatorname{mult}_{P}(D)\right| ; P \subset X, \operatorname{codim}(P, X)=1\right\} .
$$

Theorem 1.7. 2] Let $X$ be a nonsingular variety and let $\pi: X \rightarrow S$ be a proper morphism. Let $D, B$ be $\mathbb{R}$-divisors on $X$, satisfying the following properties:

(1) $D$ is nef/S.

(2) The $\mathbb{R}$-divisor $r D-(K+B)$ is nef/S and big/S, for some $r \in \mathbb{Q}$.

(3) $\operatorname{Supp}\{B\}$ is a simple normal crossings divisor and $\lfloor B\rfloor \leq 0$.

Then there exists $t_{0}, \epsilon>0$ such that $\pi_{*} \mathcal{O}_{X}(\lceil-B\rceil+\langle t D\rangle) \neq 0$ for every $t \in \mathbb{R}$ with $t \geq t_{0}$ and $\|\langle t D\rangle-t D\|<\epsilon$.

Corollary 1.8. Let $(X, B)$ be a log pair with Kawamata log terminal singularities and let $\pi: X \rightarrow S$ be a proper morphism. Let $\mathbf{D}$ be a b-nef $/ S \mathbb{R}$-b-divisor of $X$ such that the $\mathbb{R}$-b-divisor $r \mathbf{D}-\overline{K+B}$ is bnef/S and b-big/S for some $r \in \mathbb{R}$. 
Then there exists $t_{0}, \epsilon>0$ and a birational contraction $Y \rightarrow X$ such that $\pi_{*} \mathcal{O}_{X}(\lceil\mathbf{A}(X, B)+t \mathbf{D}\rceil) \neq 0$ for every $t \in \mathbb{R}$ with $t \geq t_{0}$ and $\left\|t \mathbf{D}_{Y}-\left\langle t \mathbf{D}_{Y}\right\rangle\right\|<\epsilon$.

Proof. Let $Y \rightarrow X$ be a resolution of singularities such that $\mathbf{D}=\overline{\mathbf{D}_{Y}}$, and $\operatorname{Supp}\left(B_{Y}\right) \cup \operatorname{Supp}\left(\mathbf{D}_{Y}\right)$ is included in a simple normal crossings divisor $\sum_{l} E_{l}$ on $Y$. Let $\pi^{\prime}: Y \rightarrow S$ be he induced proper morphism. In particular,

$$
\pi_{*} \mathcal{O}_{X}(\lceil\mathbf{A}(X, B)+t \mathbf{D}\rceil)=\pi_{*}^{\prime} \mathcal{O}_{Y}\left(\left\lceil-B_{Y}+t \mathbf{D}_{Y}\right\rceil\right) \text { for } t \in \mathbb{R} .
$$

By Theorem [1.7, there exist $t_{0}, \epsilon^{\prime}>0$ such that

$$
\pi_{*}^{\prime} \mathcal{O}_{Y}\left(\left\lceil-B_{Y}\right\rceil+\left\langle t \mathbf{D}_{Y}\right\rangle\right) \neq 0
$$

for $t \geq t_{0}$ and $\left\|t \mathbf{D}_{Y}-\left\langle t \mathbf{D}_{Y}\right\rangle\right\|<\epsilon_{0}^{\prime}$. Choose $\epsilon \in\left(0, \epsilon^{\prime}\right)$ and

$$
\epsilon \leq \min _{l}\left(1-\left\{\operatorname{mult}_{E_{l}}\left(B_{Y}\right)\right\}\right)
$$

Then for every $t \in \mathbb{R}$ with $\left\|t \mathbf{D}_{Y}-\left\langle t \mathbf{D}_{Y}\right\rangle\right\|<\epsilon$, we have

$$
\left\lceil-B_{Y}\right\rceil+\left\langle t \mathbf{D}_{Y}\right\rangle \leq\left\lceil-B_{Y}+t \mathbf{D}_{Y}\right\rceil \text {. }
$$

Therefore $\pi_{*}^{\prime} \mathcal{O}_{Y}\left(\left\lceil-B_{Y}+t \mathbf{D}_{Y}\right\rceil\right) \neq 0$ for $t \geq t_{0}$ and $\left\|t \mathbf{D}_{Y}-\left\langle t \mathbf{D}_{Y}\right\rangle\right\|<$ $\epsilon$.

\section{A SEMIAMPLENESS CRITERION}

Theorem 2.1. Let $(X, B)$ be a log pair, let $\pi: X \rightarrow S$ be a proper morphism and let $D$ be a nef/S $\mathbb{R}$-divisor on $X$, satisfying the following properties:

(i) $r D-(K+B)$ is nef/S and big/S, for some $r \in \mathbb{Q}$.

(ii) $\pi_{*} \mathcal{O}_{X}(\lceil\mathbf{A}(X, B)+j \bar{D}\rceil) \subseteq \pi_{*} \mathcal{O}_{X}(j D)$ for $I \mid j$, where $I$ is some positive integer.

(iii) For every point $P \in \operatorname{LCS}(X, B)$, there exists a non-zero rational function $a \in k(X)^{\times}$and there exists $q \in \mathbb{Q}$ such that the $\mathbb{R}$-divisor

$$
q(a)+D
$$

is effective in a neighborhood of $\pi^{-1}(\pi(P))$ and it does not contain $P$ in its support.

Then $D$ is a semiample/S $\mathbb{Q}$-divisor.

Proof. Step 1. There exists a positive multiple $I_{1}$ of $I$ such that

$$
\pi_{*} \mathcal{O}_{X}(m D) \neq 0 \text { for } I_{1} \mid m .
$$

Indeed, if $(X, B)$ does not have Kawamata log terminal singularities, the claim follows from (iii). If $(X, B)$ has Kawamata log terminal 
singularities, we infer by Corollary 1.8 and Lemma 1.6 that there exists a multiple $I_{1}$ of $I$ such that

$$
\pi_{*} \mathcal{O}_{X}\left(\left\lceil\mathbf{A}(X, B)+I_{1} \bar{D}\right\rceil\right) \neq 0 .
$$

By (ii), we obtain $\pi_{*} \mathcal{O}_{X}\left(I_{1} D\right) \neq 0$. The same holds for multiples of $I_{1}$.

Step 2. Let $\left(\mathbf{D}_{i}\right)_{i}$ be the characteristic sequence of the functional algebra of $X / S$

$$
\mathcal{R}_{X / S}(D)=\bigoplus_{i=0}^{\infty} \pi_{*} \mathcal{O}_{X}(i D) .
$$

By (iii), there exists a multiple $I_{2}$ of $I_{1}$ such that $\bar{D}-\mathbf{D}_{m}$ is zero above a neighborhood of $\operatorname{LCS}(X, B)$, for every $m \in I_{2} \mathbb{Z}_{>0}$. We replace $D, r$ by $I_{2} D, r I_{2}^{-1}$, respectively, so that we can assume from now that $I=I_{1}=I_{2}=1$. We will also shrink $S$ to an affine neighborhood of some fixed point of $S$, without further notice.

Step 3. Fix a positive integer $m$ such that $\mathbf{D}_{m} \neq \bar{D}$. There exists a resolution $\mu: Y \rightarrow X$ with the following properties (cf. [1], Corollary 0-3-6):

(1) $\mathbf{D}_{m}=\overline{\left(\mathbf{D}_{m}\right)_{Y}}$. Denote $F=\mu^{*} D-\mathbf{D}_{m, Y}$.

(2) $\operatorname{Supp}\left(B_{Y}\right) \cup \operatorname{Supp}(F)$ is included in a simple normal crossings divisor $\sum_{\alpha} G_{\alpha}$ on $Y$, where $K_{Y}+B_{Y}=\mu^{*}(K+B)$.

(3) $r \mu^{*} D-K_{Y}-B_{Y}-\sum_{\alpha} \epsilon_{\alpha} G_{\alpha}$ is an ample/ $S \mathbb{R}$-divisor, with $0<\epsilon_{\alpha} \ll 1$.

Let $\pi^{\prime}=\pi \circ \mu: Y \rightarrow S$ be the induced proper morphism. Define $c$ to be the largest real number such that the log pair

$$
\left(Y, B_{Y}+c F+\sum_{\alpha} \epsilon_{\alpha} G_{\alpha}\right)
$$

has log canonical singularities above $X \backslash \operatorname{LCS}(X, B)$. It follows from above that $F$ is supported over $X \backslash \operatorname{LCS}(X, B)$, hence

$$
c=\min _{\alpha} \frac{1-\operatorname{mult}_{G_{\alpha}}\left(B_{Y}\right)-\epsilon_{\alpha}}{\operatorname{mult}_{G_{\alpha}}(F)} .
$$

By perturbing the coefficients $\epsilon_{\alpha}$ if necessary, we may assume that the minimum is attained at a unique prime component of $F$, denoted by $E$. By definition, the $\mathbb{R}$-divisor

$$
B^{\prime}=B_{Y}+c F+\sum_{\alpha} \epsilon_{\alpha} G_{\alpha}-E .
$$


does not contain $E$ in its support, and $\left\lceil-B^{\prime}\right\rceil$ is effective above $X \backslash$ $\operatorname{LCS}(X, B)$. The identity

$$
(r+c m) \mu^{*} D-\left(K_{Y}+E+B^{\prime}\right)=r \mu^{*} D-K_{Y}-B_{Y}-\sum_{\alpha} \epsilon_{\alpha} G_{\alpha}+c m \mathbf{D}_{m, Y}
$$

implies that the $\mathbb{R}$-divisor $t \mu^{*} D-\left(K_{Y}+E+B^{\prime}\right)$ is ample/S for every $t \geq r+c m$.

Let $\mu^{*} D=\sum_{i} d_{i} D_{i}$ be the decomposition of $\mu^{*} D$ into prime components. There exists a $\pi^{\prime}$-very ample divisor $L$ on $Y$ such that $L+D_{i}$ is $\pi^{\prime}$-very ample for every $i$. Choose general elements $D_{i}^{1} \in\left|L+D_{i}\right|$ and $D_{i}^{2} \in|L|$ and set $D^{\prime}=\sum_{i} d_{i}\left(D_{i}^{1}-D_{i}^{2}\right)$. The following properties hold:

(4) $\mu^{*} D \sim_{\mathbb{R}} D^{\prime}$.

(5) $\left\langle t \mu^{*} D\right\rangle \sim\left\langle t D^{\prime}\right\rangle$ for every $t \in \mathbb{R}$ with $\left\|\left\langle t \mu^{*} D\right\rangle-t \mu^{*} D\right\|<1 / 2$.

(6) $\operatorname{Supp}\left(D^{\prime}\right) \cup \operatorname{Supp}(E) \cup \operatorname{Supp}\left(B^{\prime}\right)$ is included in a simple normal crossings divisor on $Y$.

There exists $\epsilon_{1}>0$ such that the $\mathbb{R}$-divisor

$$
\left\langle t D^{\prime}\right\rangle-K_{Y}-E-B^{\prime}
$$

is ample/S for $t \geq r+c m$ and $\left\|\left\langle t D^{\prime}\right\rangle-t D^{\prime}\right\|<\epsilon_{1}$. We infer by Kawamata-Viehweg vanishing (1], Theorem 1-2-3) that the restriction map

$$
\pi_{*}^{\prime} \mathcal{O}_{Y}\left(\left\lceil-B^{\prime}\right\rceil+\left\langle t D^{\prime}\right\rangle\right) \rightarrow \pi_{*}^{\prime} \mathcal{O}_{E}\left(\left\lceil-\left.B^{\prime}\right|_{E}\right\rceil+\left\langle\left. t D^{\prime}\right|_{E}\right\rangle\right)
$$

is surjective for $t \geq r+c m$ and $\left\|\left\langle t D^{\prime}\right\rangle-t D^{\prime}\right\|<\epsilon_{1}$. On the other hand, the restrictions of $\left(E,\left.B^{\prime}\right|_{E}\right)$ and $\left.D^{\prime}\right|_{E}$ to the generic fiber of $E \rightarrow \pi^{\prime}(E)$ satisfy the assumptions of Theorem 1.7. Indeed, (i) and (iii) are clear, whereas (ii) follows from the $\pi^{\prime}$-ampleness of the $\mathbb{R}$ divisor $\left.(r+c m) D^{\prime}\right|_{E}-\left(K_{E}+\left.B^{\prime}\right|_{E}\right)$. Therefore there exists $t_{0} \geq r+c m$ and $0<\epsilon_{2}<\epsilon_{1}$ such that

$$
\pi_{*}^{\prime} \mathcal{O}_{E}\left(\left\lceil-\left.B^{\prime}\right|_{E}\right\rceil+\left\langle\left. t D^{\prime}\right|_{E}\right\rangle\right) \neq 0
$$

for every $t$ with $t \geq t_{0}$ and $\left\|\left\langle t D^{\prime}\right\rangle-t D^{\prime}\right\|<\epsilon_{2}$. In particular,

$$
E \nsubseteq \mathrm{Bsl}_{\pi^{\prime}}\left|\left\lceil-B^{\prime}\right\rceil+\left\langle t D^{\prime}\right\rangle\right| \text { for } t \geq t_{0},\left\|\left\langle t D^{\prime}\right\rangle-t D^{\prime}\right\|<\epsilon_{2} .
$$

Choose $0<\epsilon_{3}<\min \left(\epsilon_{2}, \frac{1}{2}\right)$. We obtain

$$
E \nsubseteq \mathrm{Bsl}_{\pi^{\prime}}\left|\left\lceil-B^{\prime}\right\rceil+\left\langle t \mu^{*} D\right\rangle\right| \text { for } t \geq t_{0},\left\|\left\langle t \mu^{*} D\right\rangle-t \mu^{*} D\right\|<\epsilon_{3} .
$$

It is easy to see that there exists $\epsilon_{4}<\epsilon_{3}$ such that

$$
\left\lceil-B^{\prime}\right\rceil+\left\langle t \mu^{*} D\right\rangle \leq\left\lceil-B^{\prime}+t \mu^{*} D\right\rceil \text { for }\left\|\left\langle t \mu^{*} D\right\rangle-t \mu^{*} D\right\|<\epsilon_{4} .
$$

By Lemma 1.6. there exists a multiple $j$ of $m$ such that $j \geq t_{0}, \| j \mu^{*} D$ $\left\langle j \mu^{*} D\right\rangle \|<\epsilon_{4}$ and $\operatorname{mult}_{E}\left(j \mu^{*} D-\left\langle j \mu^{*} D\right\rangle\right) \leq 0$. In particular, there 
exists a rational function $a \in k(Y)^{\times}$such that the divisor

$$
(a)+\left\lceil-B^{\prime}\right\rceil+\left\langle j \mu^{*} D\right\rangle
$$

is effective and it does not contain $E$ in its support. Since $\operatorname{mult}_{E}\left(B^{\prime}\right)=$ 0 , the latter property is equivalent to

$$
\operatorname{mult}_{E}\left((a)+\left\langle j \mu^{*} D\right\rangle\right)=0 .
$$

On the other hand, the following inclusions hold

$$
\pi_{*}^{\prime} \mathcal{O}_{Y}\left(\left\lceil-B^{\prime}\right\rceil+\left\langle j \mu^{*} D\right\rangle\right) \subseteq \pi_{*}^{\prime} \mathcal{O}_{Y}\left(\left\lceil-B_{Y}+j \mu^{*} D\right\rceil\right) \subseteq \pi_{*}^{\prime} \mathcal{O}_{Y}\left(j \mu^{*} D\right) .
$$

Therefore $(a)+j \mu^{*} D \geq 0$. In particular, $\operatorname{mult}_{E}\left(j \mu^{*} D-\left\langle j \mu^{*} D\right\rangle\right) \geq 0$. The opposite inclusion holds by assumption, hence $\operatorname{mult}_{E}\left(j \mu^{*} D\right) \in \mathbb{Z}$. Therefore the effective $\mathbb{R}$-divisor $(a)+j \mu^{*} D$ has multiplicity zero at $E$. In particular, mult ${ }_{E}\left(\mathbf{D}_{j}-\bar{D}\right)=0$. By Lemma 1.3, there exists an open set $V$ in $X$ such that $V \cap \mu(E) \neq \emptyset$ and $\left.\left(\bar{D}-\mathbf{D}_{j}\right)\right|_{V}=0$.

Step 4. Let $m \geq 1$. By Lemma 1.3, there exists a closed subset $\mathrm{Bsl}_{\pi}|m D|$ of $X$ such that for every prime b-divisor $E$ of $X, \operatorname{mult}_{E}(\bar{D}-$ $\left.\mathbf{D}_{j}\right)=0$ if and only if $c_{X}(E) \subset \mathrm{Bsl}_{\pi}|m D|$. For $m \mid m^{\prime}$, we have $\mathbf{D}_{m} \leq \mathbf{D}_{m^{\prime}}$, hence $\mathrm{Bsl}_{\pi}|m D| \supseteq \operatorname{Bsl}_{\pi}\left|m^{\prime} D\right|$. From above, the following properties hold:

(1) $\mathrm{Bsl}_{\pi}|m D| \subsetneq X$ for $m \geq 1$.

(2) For every $m$, there exists $m \mid m^{\prime}$ such that $\mathrm{Bsl}_{\pi}|m D| \supsetneq \mathrm{Bsl}_{\pi}\left|m^{\prime} D\right|$.

By Noetherian induction, there exists a positive integer $m$ such that $\mathrm{Bsl}_{\pi}|m D|=\emptyset$, that is $\mathbf{D}_{m}=\bar{D}$. Therefore $D$ is rational, and the Cartier divisor $m D$ is $\pi$-free.

We can use Theorem 2.1 to reprove Kawamata's result ([2], Theorem 1) on the finite generation of the log canonical ring modulo the existence of a Cutkosky-Kawamata-Moriwaki decomposition:

Theorem 2.2. 2] Let $(X, B)$ be a log variety with Kawamata log terminal singularities such that $K+B$ is $\mathbb{Q}$-Cartier and let $\pi: X \rightarrow S$ be a proper morphism. Assume that $\mathbf{P}$ is a b-nef/S and b-big/S $\mathbb{R}$-b-divisor of $X$ such that

(1) $\mathbf{P} \leq \overline{K+B}$.

(2) $\mathcal{R}_{X / S}(\mathbf{P})=\mathcal{R}_{X / S}(K+B)$.

Then $\mathbf{P}$ is rational and b-semiample/S. In particular, the log canonical $\mathcal{O}_{S}$-algebra $\mathcal{R}_{X / S}(K+B)$ is finitely generated.

Proof. Step 1. Let $\mu: Y \rightarrow X$ be a resolution of singularities such that $\mathbf{P}=\overline{\mathbf{P}_{Y}}$. Let $\mu^{*}(K+B)=K_{Y}+B_{Y}$ be the log pullback, and let $B_{Y}=B_{Y}^{+}-B_{Y}^{-}$be the decomposition of $B_{Y}$ into its positive and 
negative part, respectively. The effective $\mathbb{Q}$-divisor $B_{Y}^{-}$is $\mu$-exceptional, and

$$
K_{Y}+B_{Y}^{+}=\mu^{*}(K+B)+B_{Y}^{-} \text {. }
$$

In particular, $\mathbf{P}_{Y} \leq K_{Y}+B_{Y}^{+}$and $\mathcal{R}_{Y / S}\left(K_{Y}+B_{Y}^{+}\right)=\mathcal{R}_{X / S}(K+B)$. Therefore $\left(Y, B_{Y}^{+}\right)$is a log variety with Kawamata log terminal singularities, $\mathbf{P} \leq \overline{K_{Y}+B_{Y}^{+}}, \mathcal{R}_{Y / S}(\mathbf{P})=\mathcal{R}_{Y / S}\left(K_{Y}+B_{Y}^{+}\right)$. Furthermore, $\mathbf{P}=\overline{\mathbf{P}_{Y}}$.

Step 2. By Step 1, we may assume that $X$ is nonsingular, $\mathbf{P}=\bar{P}$ for some $\mathbb{R}$-divisor $P$ on $X$, and if we denote $N=K+B-P$, then $\operatorname{Supp}(B) \cup \operatorname{Supp}(N) \cup \operatorname{Supp}(P)$ is included in a simple normal crossings divisor on $X$. Define $B^{\prime}=B-N$ and let $I$ be the smallest positive integer such that $I(K+B)$ is an integral divisor. For $I \mid j$, the following inequalities hold:

$$
\left\lceil j P-B^{\prime}\right\rceil \leq\lceil j P+N\rceil \leq\lceil j P+j N\rceil=j(K+B) .
$$

Since $\pi_{*} \mathcal{O}_{X}(j P)=\pi_{*} \mathcal{O}_{X}(j(K+B))$, we obtain

$$
\pi_{*} \mathcal{O}_{X}\left(\left\lceil j P-B^{\prime}\right\rceil\right) \subseteq \pi_{*} \mathcal{O}_{X}(j P) \text { for } I \mid j .
$$

Furthermore, $P=K+B^{\prime}$, hence $2 P-\left(K+B^{\prime}\right)=P$ is nef $/ S$ and $\mathrm{big} / S$. By Theorem 2.1, $P$ is rational and semiample $/ S$.

\section{Finite generation of some Algebras}

Proposition 3.1. Let $\pi: X \rightarrow S$ be a proper surjective morphism and let $D$ be a nef/S and big/S $\mathbb{R}$-divisor on $X$. Then:

(1) The characteristic limit of the $\mathcal{O}_{S}$-algebra $\mathcal{R}_{X / S}(D)$ is $\bar{D}$.

(2) The $\mathcal{O}_{S}$-algebra $\mathcal{R}_{X / S}(D)$ is finitely generated if and only if $D$ is rational and semiample/S.

(3) Let $(X, B)$ be a log pair structure on $X$, and let $\mathbf{D}$. be the characteristic sequence of $\mathcal{R}_{X / S}(D)$. Then for every $j \geq 1$, there exists a positive integer $I(j)$ such that the natural inclusion of sheaves

$$
\mathcal{O}_{X}\left(\left\lceil\mathbf{A}(X, B)+j \mathbf{D}_{i}\right\rceil\right) \subseteq \mathcal{O}_{X}(\lceil\mathbf{A}(X, B)+j \bar{D}\rceil)
$$

is an equality for $I(j) \mid i$.

Proof. If $\mu: Y \rightarrow X$ is a birational contraction, it is clear that we may replace $X$ and $D$ by $Y$ and $\mu^{*} D$, respectively. In particular, we may assume that $X$ is nonsingular and $\pi$ is projective. We may also assume that $S$ is affine.

We claim that there exists a sequence of ample $/ S \mathbb{Q}$-divisors $Q_{k}$, supported by some fixed reduced divisor on $X$, such that $Q_{k} \leq D$ and $\lim _{k \rightarrow \infty} Q_{k}=D$. Indeed, by Kodaira's Lemma, there exists an effective 
$\mathbb{R}$-divisor $E$ on $X$ such that $D-E$ is an ample $/ S \mathbb{Q}$-divisor. Since $D$ is nef/ $S$, it follows that $D-\frac{1}{k} E$ is an ample/S $\mathbb{R}$-divisor for every $k \geq 1$. Since ampleness is an open condition and $X$ is $\mathbb{Q}$-factorial, we may find for each $k$ an ample $/ S \mathbb{Q}$-divisor $D_{k}$, supported by $\operatorname{Supp}(D) \cup \operatorname{Supp}(E)$, such that $Q_{k} \leq D-\frac{1}{k} E$ and $\left\|D-\frac{1}{k} E-Q_{k}\right\| \leq \frac{1}{k}$.

(1) Consider the sequence $\left(Q_{k}\right)_{k \geq 1}$ constructed above. For each $k \geq 1$, there exists a positive integer $m_{k}$ such that $m_{k} Q_{k}$ is free/S. Since $m_{k} Q_{k} \leq m_{k} D$, we obtain $\overline{m_{k} Q_{k}} \leq \mathbf{M}_{m_{k}}$, that is $\overline{Q_{k}} \leq \mathbf{D}_{m_{k}}$. In particular,

$$
\overline{Q_{k}} \leq \mathbf{D} .
$$

Since $\lim _{k \rightarrow \infty} Q_{k}=D$, we obtain

$$
\bar{D}=\lim _{k \rightarrow \infty} \overline{Q_{k}} \leq \mathbf{D} .
$$

The opposite inequality always holds, hence $\mathbf{D}=\bar{D}$.

(2) If $\mathcal{R}_{X / S}(D)$ is finitely generated, then the characteristic limit $\mathbf{D}$ is rational and semiample/S. By (1), $D$ is rational and semiample/S. The converse is clear.

(3) If $\mu: Y \rightarrow X$ is a birational contraction, let $\mu^{*}(K+B)=K_{Y}+$ $B_{Y}$. Then $\mathbf{A}(X, B)=\mathbf{A}\left(Y, B_{Y}\right)$ and we may replace $(X, B)$ by $\left(Y, B_{Y}\right)$. In particular, using [1, Corollary 0-3-6, we may assume that $X$ is nonsingular and contains a simple normal crossings divisor supporting $B, D$ and $Q_{k}$, for every $k \geq 1$.

Fix $j \geq 1$. Since $j Q_{k} \leq j D$ and $\lim _{k \rightarrow \infty} j Q_{k}=j D$, there exists $k=k(j)$ such that

$$
\left\lceil-B+j Q_{k}\right\rceil=\lceil-B+j D\rceil .
$$

We have $\overline{Q_{k}} \leq \mathbf{D}_{m_{k}}$, hence for $m_{k} \mid i$ the following inclusion holds:

$\mathcal{O}_{X}\left(\left\lceil\mathbf{A}(X, B)+j \overline{Q_{k}}\right\rceil\right) \subseteq \mathcal{O}_{X}\left(\left\lceil\mathbf{A}(X, B)+j \mathbf{D}_{i}\right\rceil\right) \subseteq \mathcal{O}_{X}(\lceil\mathbf{A}(X, B)+j \bar{D}\rceil)$

On the other hand, by the simple normal crossings assumption, we have

$$
\mathcal{O}_{X}\left(\left\lceil\mathbf{A}(X, B)+j \overline{Q_{k}}\right\rceil\right)=\mathcal{O}_{X}\left(\left\lceil-B+j Q_{k}\right\rceil\right)
$$

and

$$
\mathcal{O}_{X}(\lceil\mathbf{A}(X, B)+j \bar{D}\rceil)=\mathcal{O}_{X}(\lceil-B+j D\rceil) .
$$

The claim holds if we set $I(j)=m_{k(j)}$.

Theorem 3.2. Let $(X, B)$ be a log pair with Kawamata log terminal singularities and let $\pi: X \rightarrow S$ be a proper morphism. Assume that $D$ is a nef/S and big/S $\mathbb{R}$-divisor on $X$ such that

(1) $\mathcal{R}_{X / S}(D)$ is $\mathbf{A}(X, B)$-asymptotically saturated.

(2) $r D-(K+B)$ is nef/S, for some $r \in \mathbb{Q}$. 
Then $\mathcal{R}_{X / S}(D)$ is a finitely generated $\mathcal{O}_{S}$-algebra.

Proof. By Proposition 3.1. (1) is equivalent to the following property:

$$
\pi_{*} \mathcal{O}_{X}(\lceil\mathbf{A}(X, B)+j \bar{D}\rceil) \subseteq \pi_{*} \mathcal{O}_{X}(j D) \text { for } I \mid j .
$$

By Theorem 2.1, $D$ is rational and semiample/S. Therefore the algebra $\mathcal{R}_{X / S}(D)$ is finitely generated.

Theorem 3.3. Let $(X, B)$ be a log pair and let $\pi: X \rightarrow S$ be a contraction. Let $\mathcal{L}$ be a bounded functional algebra of $X / S$, with characteristic sequence $\left(\mathbf{D}_{i}\right)_{i \in \mathbb{N}(\mathcal{L})}$ and characteristic limit $\mathbf{D}$. Assume that the following properties hold:

(1) $\mathcal{L}$ is asymptotically $\mathbf{A}(X, B)$-saturated.

(2) $\mathbf{D}_{i}=\overline{\mathbf{D}_{i, X}}$ for every $i \in \mathbb{N}(\mathcal{L})$.

(3) $r \mathbf{D}_{X}-(K+B)$ is nef/S and big/S, for some $r \in \mathbb{Q}$.

(4) There exists $i \in \mathbb{N}(\mathcal{L})$ and an open neighborhood $U$ of $\operatorname{LCS}(X, B)$ such that $\left.\mathbf{D}_{i}\right|_{U}=\left.\mathbf{D}\right|_{U}$.

Then $\mathcal{L}$ is a finitely generated $\mathcal{O}_{S}$-algebra.

Proof. If $\mu: Y \rightarrow X$ is a birational contraction, let $\mu^{*}(K+B)=K_{Y}+$ $B_{Y}$ be the induced crepant log pair structure on $Y$. We have $\mathbf{A}(X, B)=$ $\mathbf{A}\left(Y, B_{Y}\right)$. By (2) and (3), we have $\mathbf{D}=\overline{\mathbf{D}_{X}}$. Therefore the asumptions are preserved if we replace $(X, B)$ by $\left(Y, B_{Y}\right)$. In particular, we may assume that $X$ is nonsingular and admits a normal crossings divisor supporting $B$ and every $\mathbf{D}_{i, X}$.

By (2), we have $\mathbf{D}_{i}=\overline{\mathbf{D}_{i, X}}$ for every $i$. In particular, every $\mathbb{Q}$-divisor $\mathbf{D}_{i, X}$ is nef $/ S$ and their limit $D=\lim _{i \rightarrow \infty} \mathbf{D}_{i, X}$ is a nef $/ S \mathbb{R}$-divisor. By the normal crossings assumption and (2), asymptotic saturation is equivalent to the following inclusions

$$
\pi_{*} \mathcal{O}_{X}\left(\left\lceil-B+j \mathbf{D}_{i, X}\right\rceil\right) \subseteq \pi_{*} \mathcal{O}_{X}\left(\mathbf{M}_{j}\right) \text { for } I \mid i, j .
$$

For each $j$, we have $\left\lceil-B+j \mathbf{D}_{i, X}\right\rceil=\lceil-B+j D\rceil$ for $i$ sufficiently large and divisible. Therefore

$$
\pi_{*} \mathcal{O}_{X}(\lceil-B+j D\rceil) \subseteq \pi_{*} \mathcal{O}_{X}\left(\mathbf{M}_{j}\right) \subseteq \pi_{*} \mathcal{O}_{X}(j D) \text { for } I \mid j .
$$

Since $D-\mathbf{D}_{i, X}$ is effective and zero on the open neighborhood $U$ of $\operatorname{LCS}(X, B)$, and $i \mathbf{D}_{i, X}$ is free/S, the $\mathbb{R}$-divisor $i D$ is free/S on $U$. Furthermore, $r D-(K+B)$ is nef $/ S$ and big/S. Theorem 2.1 implies that $D$ is a semiample/ $S \mathbb{Q}$-divisor.

Let $j$ be a multiple of $I$ such that $j D$ is free/S. There exists a contraction $\Phi: X \rightarrow T$, defined over $S$, and an ample/S Cartier divisor $A$ on $T$ such that $j D \sim \Phi^{*}(A)$. 
Set $C=\left\lceil-B^{\geq 1}\right\rceil \leq 0$, so that $\operatorname{LCS}(X, B)=\operatorname{Supp}(C)$. By assumption, $\mathbf{D}_{j}=\mathbf{D}$ over the neighborhood $U$ of $\operatorname{Supp}(C)$. By Lemma 1.4. there exist an open set $V_{j} \supset \Phi(\operatorname{Supp}(C))$ such that

$$
\left.\mathbf{D}_{j}\right|_{\Phi^{-1}\left(V_{j}\right)}=\left.\mathbf{D}\right|_{\Phi^{-1}\left(V_{j}\right)} \text {. }
$$

If $\Phi(\operatorname{Supp}(C))=T$, then $V_{j}=T$ and we are done. Assume now that $\Phi(\operatorname{Supp}(C)) \varsubsetneqq T$.

Since $A$ is ample/S, we may replace $j$ by a multiple, so that the subsheaf

$$
\Phi_{*} \mathcal{O}_{Y}(C) \otimes \mathcal{O}_{T}(j A) \subset \mathcal{O}_{T}(j A)
$$

is relatively generated by global sections. In particular, the divisor $\lceil C+j D\rceil$ is free on $Y \backslash \Phi^{-1} \Phi(\operatorname{Supp}(C))$. By asymptotic saturation, we have

$$
\pi_{*} \mathcal{O}_{X}(\lceil C+j D\rceil) \subseteq \pi_{*} \mathcal{O}_{X}(\lceil-B+j D\rceil) \subseteq \pi_{*} \mathcal{O}_{X}\left(\mathbf{M}_{j}\right) .
$$

Therefore $\overline{j D} \leq j \mathbf{D}_{j}$ above $X \backslash \Phi^{-1} \Phi(\operatorname{Supp}(C))$. The opposite inclusion always holds, hence $\mathbf{D}_{j}=\mathbf{D}$ over $X \backslash \Phi^{-1} \Phi(\operatorname{Supp}(C))$. The two open sets $\Phi^{-1}\left(V_{j}\right)$ and $X \backslash \Phi^{-1} \Phi(\operatorname{Supp}(C))$ cover $X$, hence we obtain $\mathbf{D}_{j}=\mathbf{D}$.

Therefore the $\mathcal{O}_{S}$-algebra $\mathcal{L}$ is finitely generated.

\section{REFERENCES}

[1] Kawamata, Y.; Matsuda, K.; Matsuki, K., Introduction to the minimal model problem. Algebraic geometry, Sendai, 1985, 283-360, Adv. Stud. Pure Math., 10, North-Holland, Amsterdam, 1987.

[2] Kawamata, Y., The Zariski decomposition of log-canonical divisors, Algebraic geometry, Bowdoin, 1985, 425-433, Proc. Sympos. Pure Math., 46, Part 1, Amer. Math. Soc., Providence, RI, 1987.

[3] Shokurov, V. V., Three-dimensional log perestroikas. (Russian) With an appendix in English by Yujiro Kawamata. Izv. Ross. Akad. Nauk Ser. Mat. 56 (1992), no. 1, 105-203; translation in Russian Acad. Sci. Izv. Math. 40 (1993), no. 1, 95-202.

[4] Shokurov, V. V., 3-fold log models. Algebraic geometry, 4. J. Math. Sci. 81 (1996), no. 3, 2667-2699.

[5] Shokurov, V. V., Prelimiting flips. Tr. Mat. Inst. Steklova 240 (2003), Biratsion. Geom. Linein. Sist. Konechno Porozhdennye Algebry, 82-219; translation in Proc. Steklov Inst. Math. 2003, no. 1 (240), 75-213.

RIMS, Kyото University, KyоTo 606-8502, JAPAN.

E-mail address: ambro@kurims.kyoto-u.ac.jp 